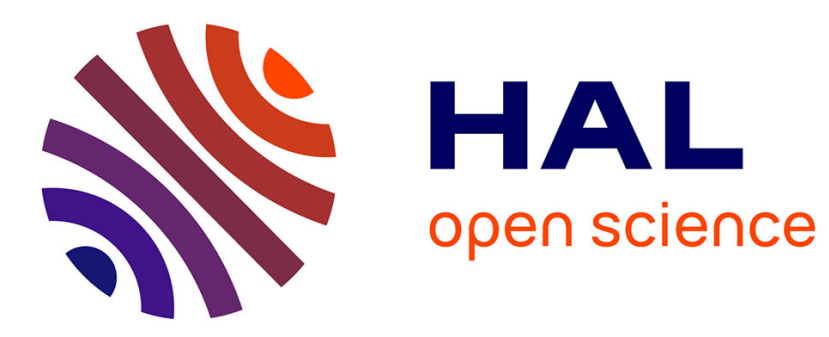

\title{
Bulletin du Commerce des bois
}

- [.]revue Forestière Française, Rédaction

\section{- To cite this version:}

- [.]revue Forestière Française, Rédaction. Bulletin du Commerce des bois. 1961, pp.75.

10.4267/2042/24319 . hal-03535536

\section{HAL Id: hal-03535536 \\ https://hal.science/hal-03535536}

Submitted on 19 Jan 2022

HAL is a multi-disciplinary open access archive for the deposit and dissemination of scientific research documents, whether they are published or not. The documents may come from teaching and research institutions in France or abroad, or from public or private research centers.
L'archive ouverte pluridisciplinaire HAL, est destinée au dépôt et à la diffusion de documents scientifiques de niveau recherche, publiés ou non, émanant des établissements d'enseignement et de recherche français ou étrangers, des laboratoires publics ou privés. 


\section{Bulletin du Commerce des Bois}

\section{Quillan}

Les ventes de 1'Inspection ont eu lieu à Quillan le 26 octobre.

L'assistance était très nombreuse: habitués du Département et des Départements voisins, et quelques exploitants inconnus.

Le volume offert $\left(47173 \mathrm{~m}^{3}\right)$ était légèrement inférieur à celui de l'exercice précédent $\left(52051 \mathrm{~m}^{3}\right)$.

Sur 114 articles offerts, 22 sont restés invendus.

I1s représentent $9 \%$ en volume œuvre-industrie.

Ce sont des lots médiocres (produits d'éclaircie et bois de feu) et mal situés.

Le volume vendu se répartit comme suit:

Bois d'cuvre. - Résineux: $27294 \mathrm{~m}^{3}$ (Sapin $95 \%$, Pin $4 \%$, Epicéa 1\%). Feuillus: $3331 \mathrm{~m}^{3}$ (Hêtre $100 \%$ ).

Bois d'industrie. - Résineux: $3910 \mathrm{~m}^{3}$ (Sapin, $87 \%$, Pin $6 \%$, Epicéa

$7 \%$ ). - Feuillus: $1246 \mathrm{~m}^{3}$ (Hêtre $100 \%$ ).

Bois de feu. - Feuillus 8080 stères (Hêtre $100 \%$ ).

$\mathrm{La}$ recette totale (charges et taxes forfaitaire comprises) atteint 2371.768 NF.

Prix des bois sur pied (charges et taxe forfaitaire comprises):

1) Prix moyens Bois d'cuvre:

$$
\begin{aligned}
& \text { Sapin } \ldots \ldots \ldots \ldots \ldots \ldots \text {. } 78,40 \mathrm{NF} \text { (hausse } 30 \% \text { ) } \\
& \text { Hêtre } \ldots \ldots \ldots \ldots \ldots \ldots . .26,08 \mathrm{NF} \text { (hausse } 47 \% \text { ) } \\
& \text { Pin } \ldots \ldots \ldots \ldots \ldots \ldots \ldots .62 \text {. } 35,62 \text { (hausse } 2 \% \text { ) }
\end{aligned}
$$

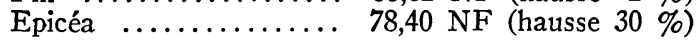

Les prix extrêmes du Sapin ont oscillé suivant la qualité des bois et les difficultés d'exploitation entre $32,40 \mathrm{NF}$ et $115,20 \mathrm{NF}$.

2) Prix moyens Bois d'Industrie:

$$
\begin{aligned}
& \text { Résineux } \ldots \ldots \ldots \ldots \ldots \\
& \text { Feuillus } \ldots \ldots \ldots \ldots \ldots \\
& 16,43
\end{aligned}
$$

3) Bois de feu: M'évente totale.

Sauf pour le Pin, dont les utilisations locales sont limitées (caisserie pour FORMICA), la hausse est très importante.

Cette tendance s'est manifestée dès le début des ventes par une concurrence très vive et une atmosphère tendue, mais sans incident.

La hausse est pratiquement constante sur toutes les coupes, quelles que soient leur qualité et leur situation, ce qui traduit une demande sensiblement supérieure à l'offre.

Les exploitants ont reçu d'importantes commandes de sciages en septembre et octobre.

Ils espèrent que cette reprise du marché sera durable et provoquera un relèvement des prix.

Ils ont acheté très cher pour faire tourner leur scierie et donner du travail à leurs ouvriers.

Bien que le volume offert corresponde sensiblement à la production des forêts, les scieries de la région travaillent seulement à $40 \%$ environ de leur capacité de production, et les « clients 》 deviennent de plus en plus nombreux, avec l'apparition des acheteurs étrangers.

Les cours pratiqués traduisent le déséquilibre du marché.

Ils sont incompatibles avec les cours actuels des grumes et des sciages.

BrousSON. 


\section{Meurthe-et-Moselle}

Les résultats des grandes Ventes de l'Exercice 1960 confirment la circonspection avec laquelle nous avions considéré ceux de 1959, touchant l'interprétation possible des prix moyens unitaires par essences et catégories.

Les facteurs d'imprécision n'ont fait que s'accentuer par la complexité accrue des éléments en cause et une certaine extravagance dans les transactions qui ont porté sur un volume sensiblement comparable à celui de l'an dernier.

C'est donc avec une solide réserve que nous avançons les prix ci-dessous :

Chêne: le $\mathrm{m}^{3}: 140-52-11 \mathrm{NF}$ (hausse moyenne $15 \%$ imputable en majeure partie à la $1^{\text {re }}$ qualité).

Hêtre: le $\mathrm{m}^{3}: 120-30 \mathrm{NF}$ (hausse moyenne $33 \%$, deux fois plus forte sur la $1^{\text {re }}$ qualité que sur la $2^{\mathrm{e}}$ ).

Résineux: le $\mathrm{m}^{3}: 81-37 \mathrm{NF}$ (hausse moyenne $16 \%$, presque toute sur la $1^{\text {re }}$ qualité).

Feuillus divers: $27-16 \mathrm{NF}$ (hausse moyenne $20 \%$ ).

Industrie: Résineux $23-$ Feuillus 13 (hausse moyenne 10 et $15 \%$ ).

Chauffage, le stère: Feuillu 2,70 NF (hausse $20 \%$ ).

1 - Observations particulières pour chacun des quatre services:

a) Briey: Absence inhabituelle de tout acheteur belge et amélioration très nette des possibilités 'd'écoulement du bois dit de feu, jusqu'ici d'un placement aléatoire dans la région industrielle.

b) Toul: Hausse accentuée tenant à l'offre quasi nulle en hêtre, hausse qui a ainsi profité artificiellement à tous les produits, même de médiocre qualité.

c) Nancy: A vu les pointes extrêmes de hausse singulièrement sur le Hêtre, donnant à la vente une allure paradoxale par la conjonction de l'incohérence apparente et de la sérénité dans l'obstination aux excès.

d) Lunéville: Surprise en sens inverse. Le flot s'est étalé à un niveau de hausse relativement faible, alors que la rumeur publique laissait, dans le secteur résineux, attendre des lames de fond.

\section{2-Observations d'ensemble:}

Cette année, le Hêtre a repris une ascension qui s'était ralentie. Le Chêne a suivi fermement avec des pointes dans la catégorie tranchage. Les résineux (sapin-épicéa) n'ont participé à l'élan général de hausse que dans une mesure très modeste, demeurant loin en arrière des prix pratiqués dans les secteurs vosgiens voisins. Il semble que pour eux seuls le facteur qualité ait joué, desservi par les soupçons persistants de mitraille.

Ailleurs et dans l'ensemble des feuillus, les acheteurs n'ont pas eu l'air de tenir sérieusement compte de ces défectuosités.

Et l'on ne peut avancer, comme l'an dernier, que ce sont les gros bois sains qui ont seuls réglé le marché.

Des produits notoirement inférieurs en Hêtre et Chêne, réellement mitraillés, ont atteint des prix de bois sains.

Il en va de même des chablis résineux qui, vendus en fin de séance, ont approché les prix de bois normaux.

On a l'impression que l'on tend vers le criterium « Masse 》 qui, fort logiquement d'ailleurs dans notre région, semble l'emporter sur celui de "Qualité 》, ce qui laisserait à penser que le sciage est en voie d'irréversible régression. C'est là un choc en retour des procédés modernes de transformation: tranchage, déroulage, trituration... qui suivent de plus près les progrès techniques et les impératifs économiques. Tous procédés également, qui, joints à des possibilités élargies d'exportation, permettent des réalisations plus immédiates allégeant les trésoreries. 
A noter enfin, comme caractère particulier à cet exercice, une reprise marquée des petits bois résineux et des qualités inférieures feuillues, ces dernières bénéficiant d'un courant accéléré vers les bois papetiers. C'est ainsi que sur le « chauffage 》 estimé (chêne excepté), l'on peut compter que $20 \%$ au moins des Quartier et Rondin sont à reconvertir en bois de cellulose, ce qui, pour la Conservation représenterait un virement de quelque 15000 stères du premier sur les seconds.

Il n'est pas douteux qu'à la lumière de ces résultats il $\mathrm{y}$ aura à l'avenir intérêt à tenir compte de cette particularité dans la classification et l'estimation des produits.

E. P. 